\title{
Intramedullary Canal-creation Technique for Patients with Osteopetrosis
}

\author{
Jonathan Kent ${ }^{1}$, David Ferguson ${ }^{2}$
}

\begin{abstract}
Aim: We present details of a surgical technique to create an intramedullary canal to allow intramedullary fracture fixation in patients with osteopetrosis. Clinical cases are used to facilitate the description.

Background: Osteopetrosis is a rare, hereditary condition characterised by hard, brittle, "marble bone;" primarily due to osteoclast dysfunction. Patients are prone to fractures and subsequently nonunions, periprosthetic fractures, and metal-ware failure are commonly seen. Due to the increased bone density, deformity, and obliteration of the medullary cavity, fracture fixation is also technically demanding.

Technique: Creation of a medullary canal allows the use of intramedullary fixation rather than plate and screws for long-bone fractures. Key factors:

- A new sharp drill bit should be used for each case as blunt drills are more likely to break.

- Bone is drilled in a pulsatile fashion, with withdrawal every 2-4 seconds for bone swarf to be removed.

- Constant cooling of the drill bit with saline to help prevent bone necrosis and drill breakage.

- Regular exchanging of drill bit sizes to expand the canal. The smaller drills start the canal and are used to direct progress. Sequential expansion during canal creation is preferred.

- Regular use of orthogonal radiographs to ensure correct canal positioning and prevent perforation.

Conclusion: The creation of an intramedullary canal allows intramedullary fracture fixation. In our experience, this technique gives the orthopaedic surgeon a safe and effective method for treating long-bone fractures in patients with osteopetrosis.

Clinical relevance: Fractures and nonunions in patients with osteopetrosis are difficult to manage; and by detailing this technique, a further option is now available for surgeons when deciding upon fixation method.

Keywords: Fracture, Intramedullary, Orthopaedics, Osteopetrosis, Pycnodysostosis, Technique.

Strategies in Trauma and Limb Reconstruction (2019): 10.5005/jp-journals-10080-1424
\end{abstract}

\section{BACKGROUND}

Osteopetrosis is a rare, hereditary condition characterised by hard, brittle, "marble bone" primarily due to osteoclast dysfunction.

The incidence is 1 in 100,000 to $500,000,{ }^{1,2}$ and it occurs in three main forms, namely, infantile, intermediate, and adult onset. These differ by their genetic inheritance and subsequent clinical findings. ${ }^{3}$ Infantile is autosomal recessive (AR) and results in bone marrow failure and a significantly decreased life expectancy unless bone marrow transplant is successfully performed. Intermediate is also due to AR inheritance, whereas adult onset has an autosomal dominant pattern and forms a spectrum of severity and clinical findings, including specific orthopaedic manifestations.

The primary underlying defect in all types of osteopetrosis is failure of bone reabsorption. This can occur due to a defect within the osteoclast lineage resulting in decreased acid secretion or osteoclast maturation or with the mesenchymal cells necessary for their correct function. Multiple genes have thus been implicated and there is also evidence of not only osteoclast involvement but also osteoblasts. ${ }^{4}$

Dysfunctional osteoclast activity is predominantly due to defective chloride channel 7 (CICN7) and T cell immune regulator 1 (TCIRG1) (116 kD subunit of vacuolar proton pump) genes. ${ }^{5}$ These result in impairment of acidification due to defective proton pumps leading to decreased acid secretion and subsequent bone reabsorption. In addition, defects to receptor activating
1,2Department of Trauma and Orthopaedics, James Cook University Hospital, Middlesbrough, United Kingdom

Corresponding Author: David Ferguson, Department of Trauma and Orthopaedics, James Cook University Hospital, Middlesbrough, United Kingdom, Phone: +44 (0)7983 516602, e-mail: david.ferguson5@nhs.net How to cite this article: Kent J, Ferguson D. Intramedullary Canalcreation Technique for Patients with Osteopetrosis. Strategies Trauma Limb Reconstr 2019;14(3):155-162.

Source of support: Nil

Conflict of interest: None

NF-K B ligand (RANKL) along with monocyte-macrophage-colonystimulating factor (M-CSF) genes are responsible for inhibiting the maturation of functional, osteoclast formation. ${ }^{4}$ Due to the clinical spectrum seen, additional genetic and environmental determinants affecting gene penetrance have also been implicated.

Other specific forms also exist, which are characterised by renal tubular acidosis and cerebral calcification due to carbonic anhydrase isoenzyme II deficiency, which again results in decreased acid secretion, ${ }^{6}$ and due to cathepsin $\mathrm{K}$ mutation resulting in pycnodysostosis.

Classical radiographic findings include "rugger jersey" spine, endobones (bone in bone), uniform bone density without corticomedullary demarcation, coxa vara, long-bone 
bowing, and broadened metaphyses. ${ }^{3}$ Up to $40 \%$ of patients experience recurrent fractures due to the altered bone matrix and biomechanical characteristics. Other findings include cranial nerve entrapment and osteomyelitis of the mandible.

Due to the abnormal bone structure and remodeling, fracture management can be complicated. The early stages of bone healing display a normal process; however, appropriate remodeling does not occur. This results in a prolonged healing stage and an area without haversian systems. ${ }^{8}$ Bone union takes approximately 1 year and as such metalwork failure as well as nonunion are common occurrences.

Due to increased bone density, deformity, and obliteration of the medullary cavity, fracture fixation is also highly demanding. Fixation needs to be strong enough to withstand a prolonged period of fracture healing, and commonly used methods such as intramedullary nailing are often not technically possible. Equipment breakages as well as blunting can occur intraoperatively leading to the use of specific instruments, such as diamond-tipped drills with regular changes, and special attention needs to be applied to the cooling of instruments to help prevent subsequent bone necrosis. ${ }^{5}$

\section{TeChNIQUe}

Patients with osteopaetrosis often do not have an intramedullary canal. This limits the orthopaedic surgeons' options for operative fixation. The creation of a medullary canal allows the use of an intramedullary device rather than plate and screw fixation for long-bone fractures.

We present a technique and a series of four cases where intramedullary nails have been utilised for fracture fixation and the technique utilised to achieve this.

The main consideration is the creation of an adequate intramedullary canal. This is achieved with careful and sequential drilling and reaming, without the use of special and expensive diamond-tipped drills. This then allows the use of an intramedullary device. We have used rush rods, titanium elastic nails, and ulna nails to attain fracture fixation.

The key factors to create an intramedullary canal are:

- A new sharp drill bit should be used for each case as blunt drills are more likely to break.

- Bone is drilled in a pulsatile fashion, with withdrawal every 2-4 seconds for bone swarf to be removed (Fig. 1).

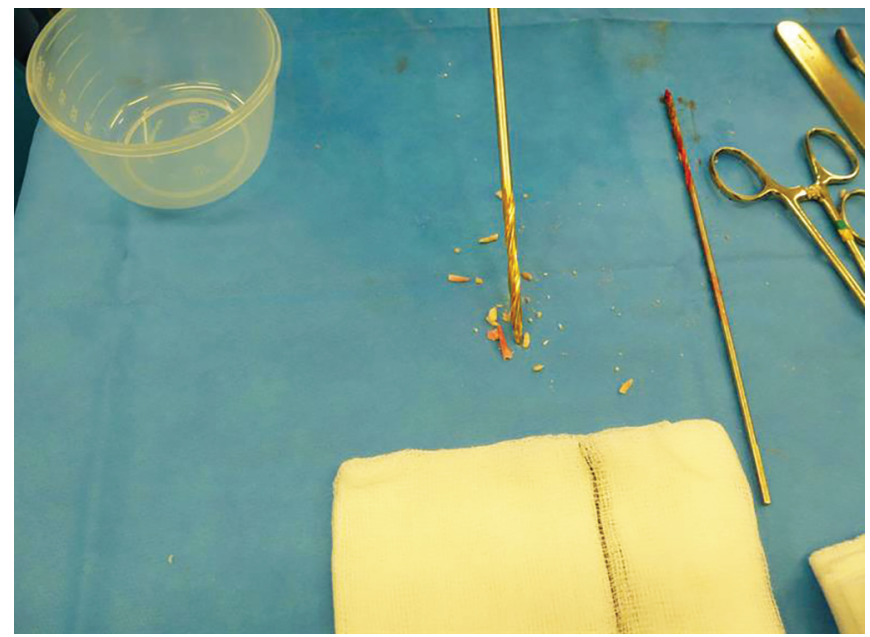

Fig. 1: Cement-like swarf removed from drill fluting. Note the $2.5 \mathrm{~mm}$ and $3.2 \mathrm{~mm}$ drill bits
- Constant cooling of the drill bit with saline to help prevent bone necrosis and drill breakage.

- Regular exchanging of drill bit sizes to expand the canal-the smaller drills start the canal and are used to direct progress. As the canal is created, sequential expansion is preferred.

- Regular use of orthogonal radiographs to ensure correct canal positioning and prevent perforation.

Unlike normal bone, osteopetrosis bone forms a powder and becomes cement like on the flutes of the drill bit (Fig. 1). This causes friction, and unless the drill is withdrawn and cleaned regularly, friction heat will burn the bone or break the drill. Saline should be constantly used to help keep the drill bit cool. A new sharp drill bit should be used for each case as the blunt drills are more likely to break. Different-sized steel drill bits should be made available. Our preference is to use $2.5 \mathrm{~mm}, 3.2 \mathrm{~mm}$, and $4.8 \mathrm{~mm}$ diameter drill bits, depending on the size of patient and the bone involved.

\section{Case Description}

\section{Case 1}

A 10-year-old boy had gradual onset of pain in his left proximal femur. He had previously sustained multiple fractures. He was diagnosed with a left proximal femoral stress fracture and was managed at another institution with a period of nonweight-bearing (Fig. 2). Despite this, his left hip gave way 1 day and a partial stress fracture progressed and became a complete fracture. The child was then transferred to our centre for further management. A computed tomography (CT) scan performed showed the remnant of a medullary canal proximally, which disappeared in the proximal one third of the diaphysis (Figs 3 and 4). This was, however, not clinically evident while performing surgery (Figs 5 and 6). An intramedullary canal was created utilising the key considerations previously described as well as the flexible hand reamers. A large stainless steel rush rod was inserted (Fig. 7). In order to control rotation, a hip spica cast was applied for 12 weeks. The mechanical axis was partially corrected at the time of surgery, with the aim to improve healing and reduce the risk of refracture (Fig. 8).

\section{Case 2}

A 16-year-old boy fell while playing basketball and sustained a fracture of his left tibia and a periprosthetic fracture of his right forearm. The tibia was managed with a circular frame and the

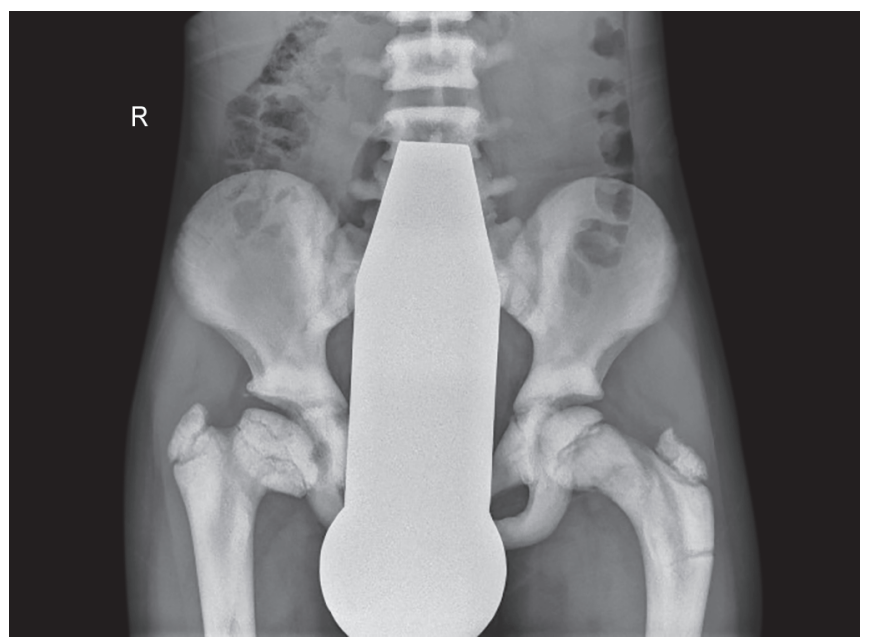

Fig. 2: A radiograph showing a partial stress fracture of the left proximal femur 


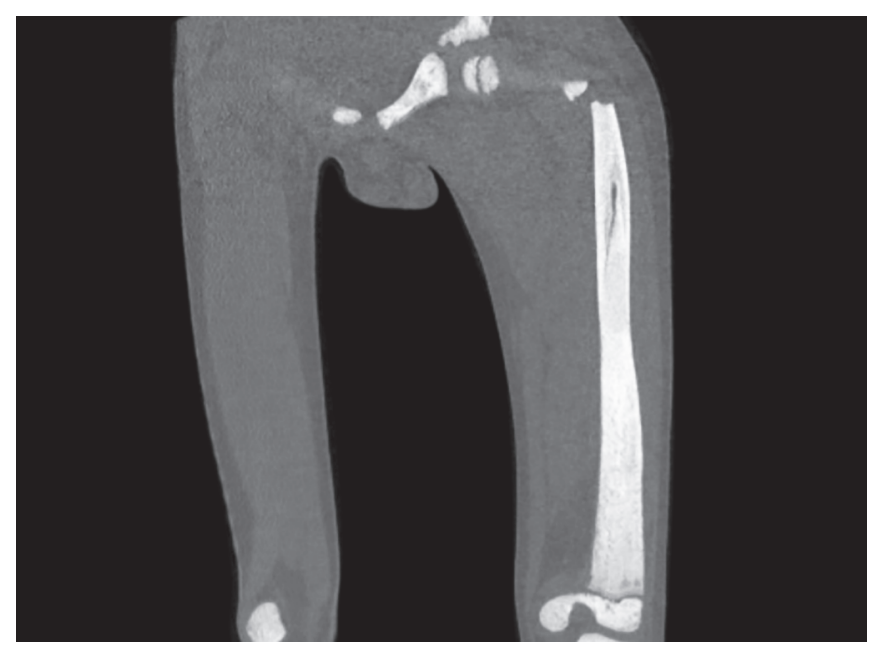

Fig. 3: A computed tomography scan was performed to check for any residual medullary canal in the left femur. A small remnant of a medullary canal was identified but this was not clinically evident while performing surgery

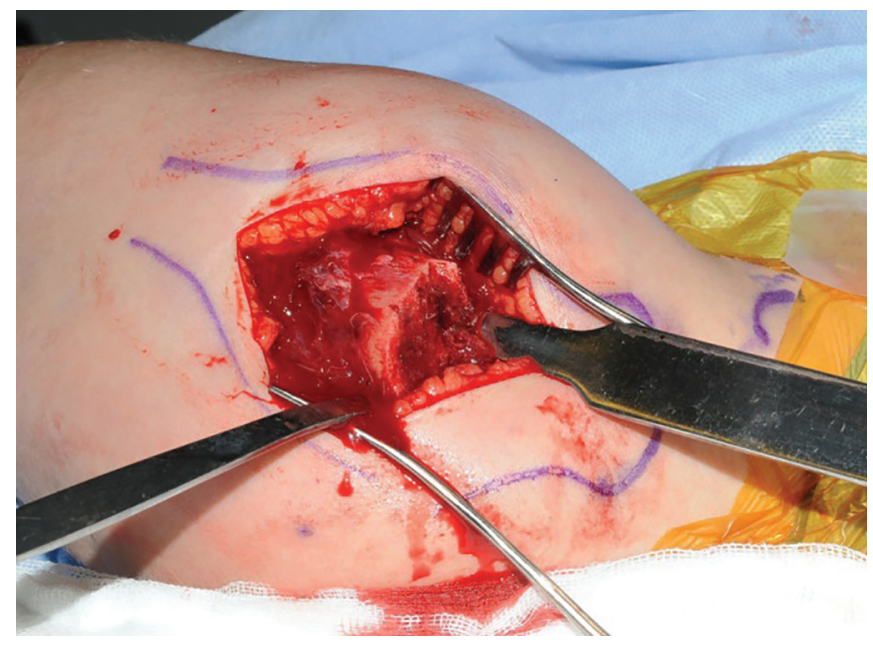

Fig. 5: Proximal femur exposed—no intramedullary canal evident

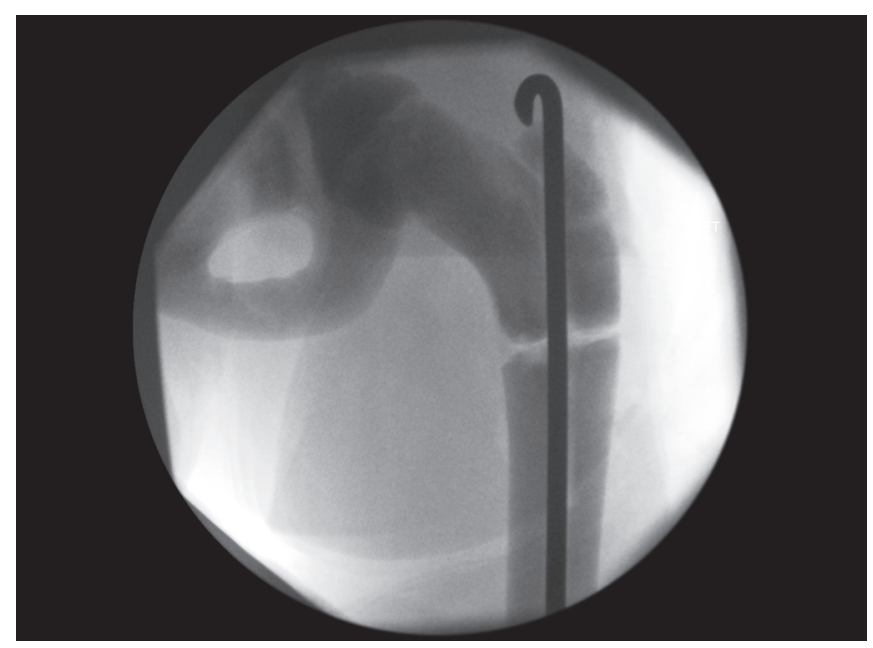

Fig. 7: Rush rod insertion from the tip of the femur. Some of the femoral neck varus has been corrected in order to minimise the risk of further stress fractures and to promote bone healing

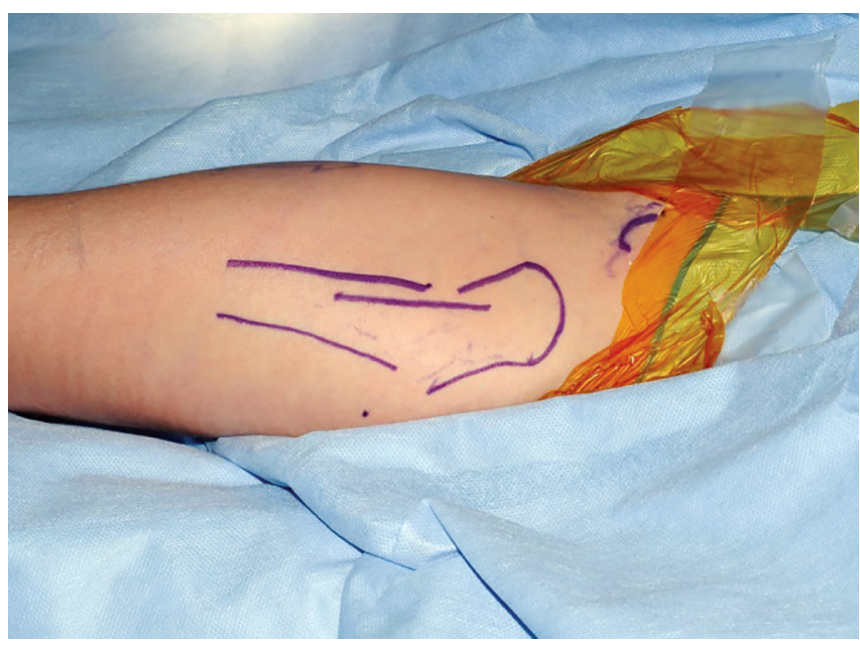

Fig. 4: Preoperative marking of fracture

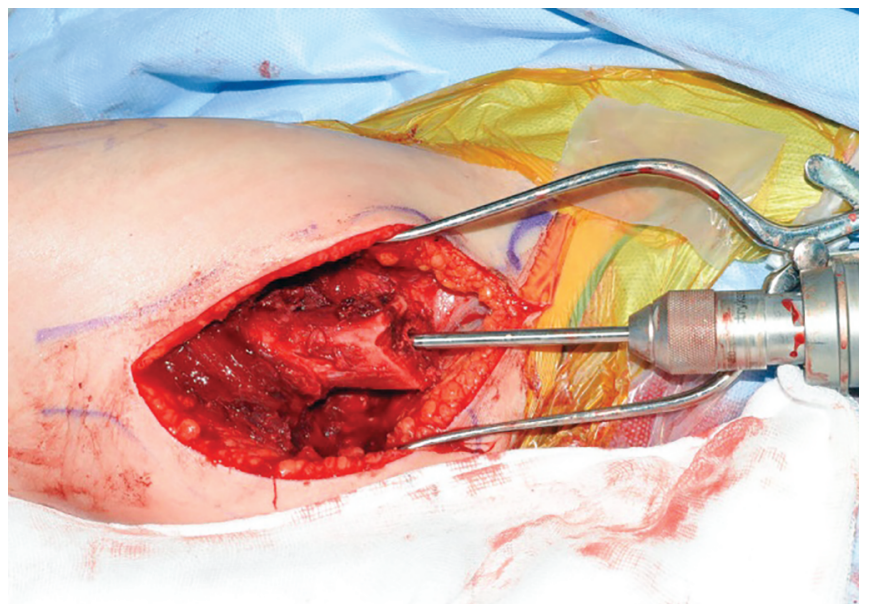

Fig. 6: Sequential drilling to create an intramedullary canal

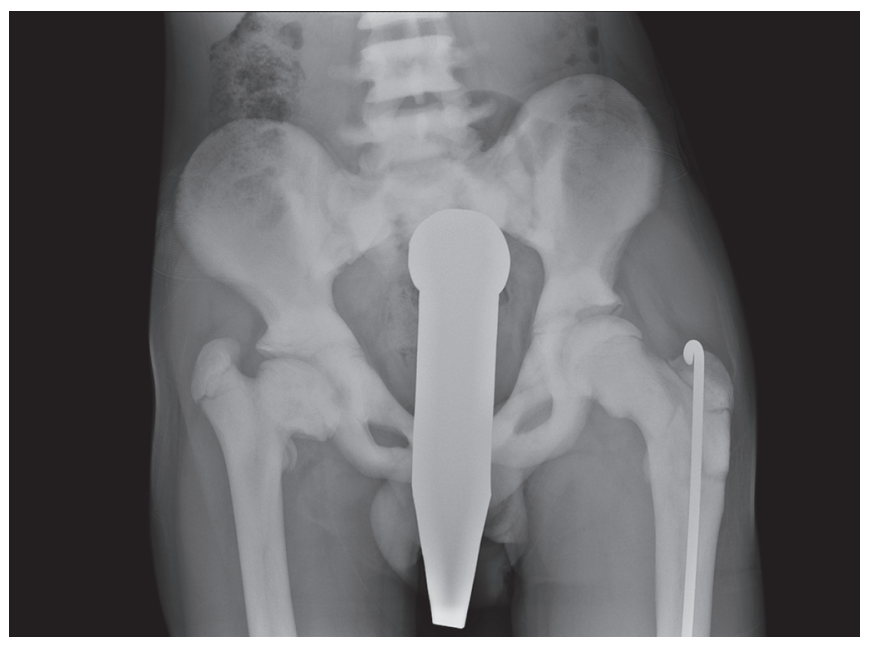

Fig. 8: Two years after reconstructive surgery. There is no evidence of avascular necrosis in the left hip and improved head-neck angle remains 


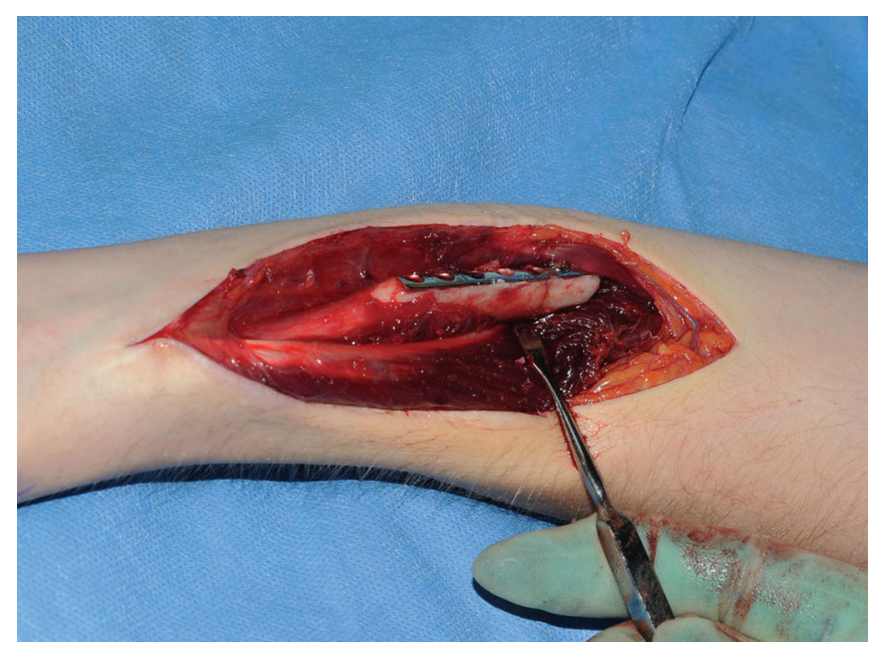

Fig. 9: The ulna fracture and broken plate is exposed. Note how there is a secondary deformity (apex anterior) in the radius, distal to the plate. This was corrected by an osteotomy and then stabilised with a titanium elastic nail

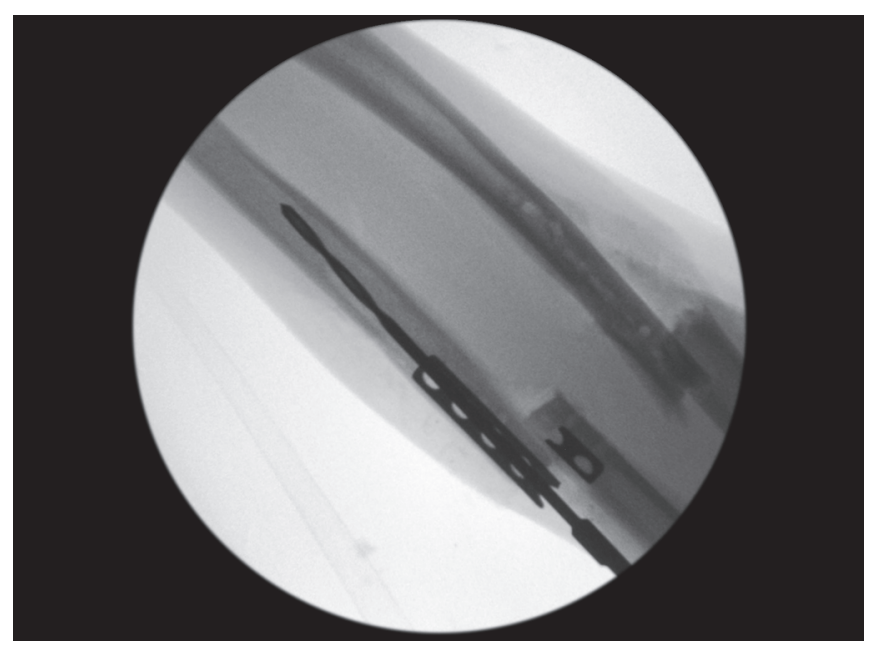

Fig. 11: Radiograph showing retained broken metal ware and drill creating intramedullary canal

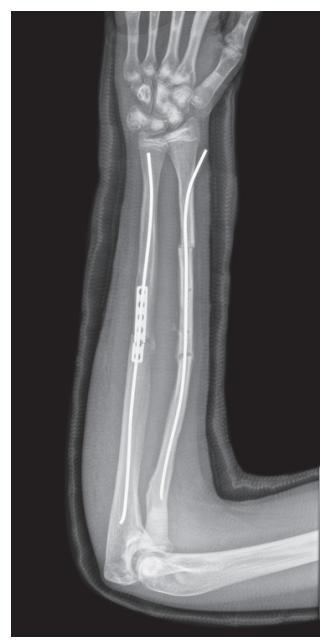

Fig. 13: Postoperative radiograph with titanium elastic nails (TEN nail, DePuy Synthes), in situ

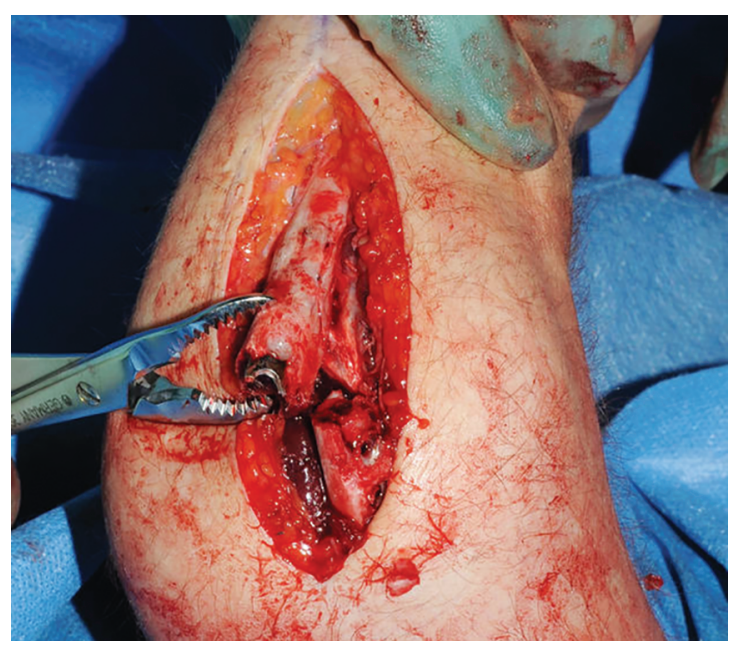

Fig. 10: The ulna shows the old third tubular plate completely enveloped in bone. Removing this would have resulted in significant bone loss

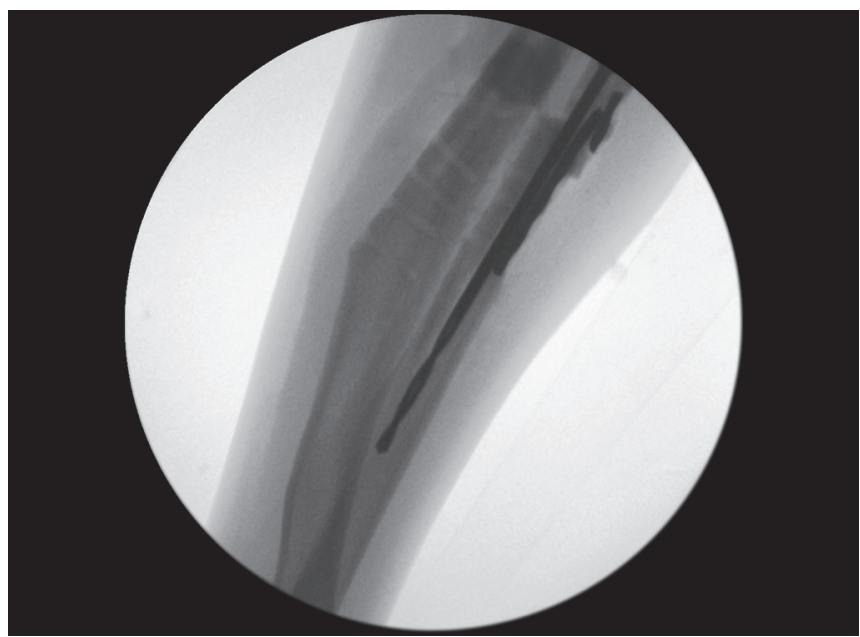

Fig. 12: Further drilling of the intramedullary canal in the ulna

forearm was fixed with intramedullary titanium elastic nails. Full union was achieved for both upper and lower limbs; however, further surgery was required due to metalwork migration.

Previous incisions were utilised to approach the fracture (Fig. 9). The broken plate on the ulna was left in situ due to it being encased in bone, but all the screws were removable (Fig. 10). An intramedullary canal was then created using the combination drilling technique, i.e., regularly switching between $2.5 \mathrm{~mm}$ and $3.2 \mathrm{~mm}$ drill bits.

It is important to continuously check with radiographs in two orthogonal planes that the drill is as central as possible (Figs 11 and 12). If the drill starts to diverge from the midline, then a smaller drill is used to create a new pilot hole to redirect the larger drill. The radius shows a significant deformity that required a corrective osteotomy prior to distal canal creation and nail insertion. By performing the corrective osteotomy, the length of drill insertion was shortened, decreasing the risk of breakage and improving the accuracy of canal creation in the distal segment as we were able to insert the drills directly into it. Titanium elastic nails were then inserted and an above elbow cast applied (Fig. 13). 


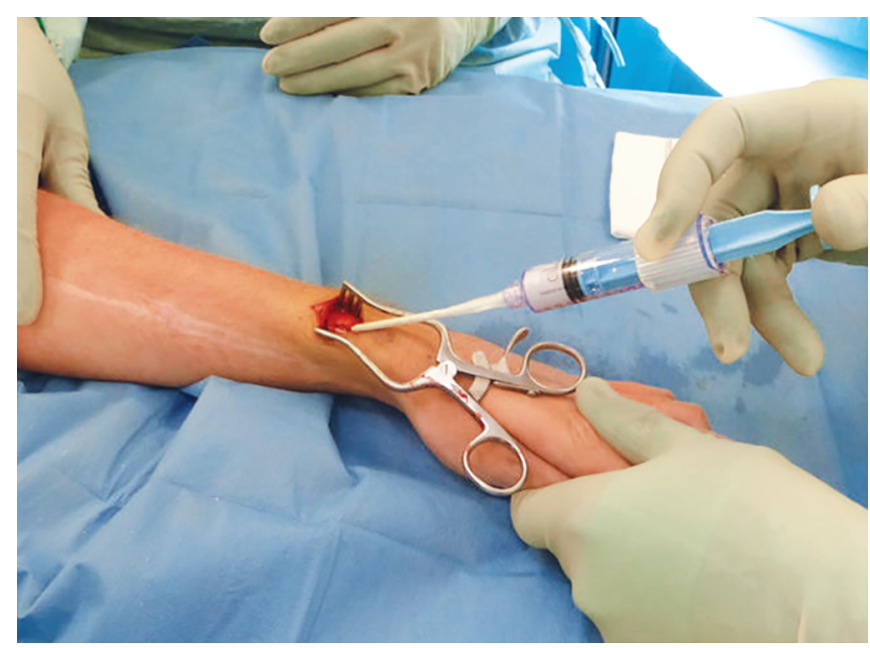

Fig. 14: Injection of cerament into titanium elastic nail exit holes after further trimming due to late migration once the fractures had healed

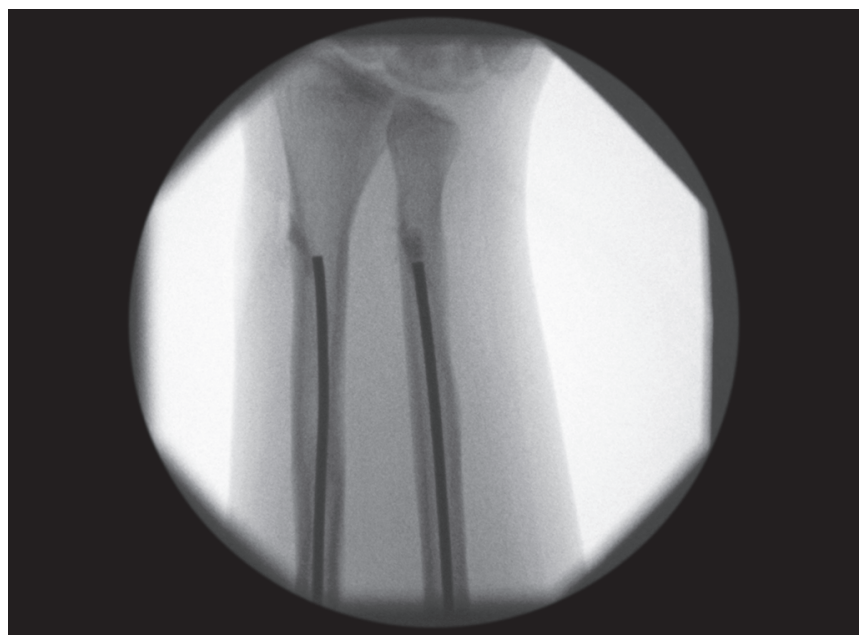

Fig. 15: Radiograph showing the titanium elastic nails buried inside the bone and the exit holes plugged by cerament bone substitute
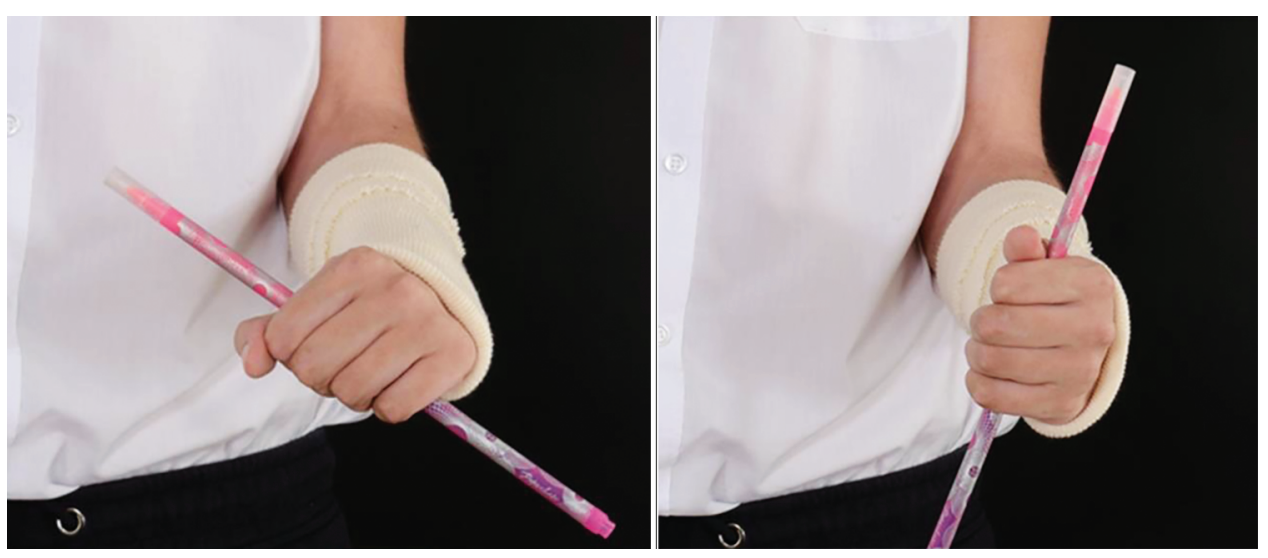

Fig. 16: Clinical photograph at 1-year follow-up showing maximum pronation (left) and supination (right)

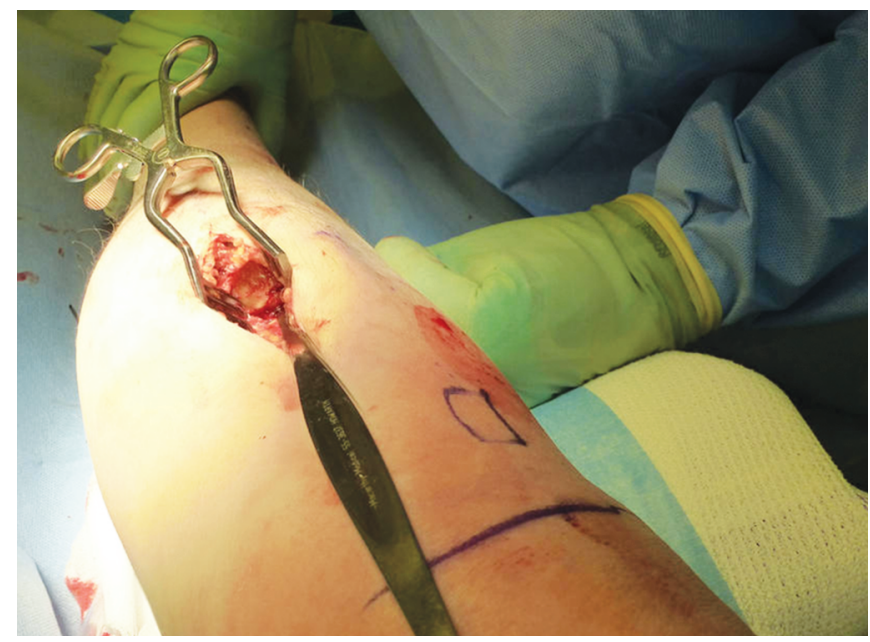

Fig. 17: The right tibia fracture has been exposed to perform the antegrade and retrograde drilling in order to create an intramedullary canal

Over the following 18 months, the titanium elastic nails began to migrate and cause some soft tissue irritation. It was deemed beneficial to retain the metalwork and so the excess material was trimmed and impacted back into the bone. The nail exit holes then had a cap of bone substitute (Cerament, Bonesupport AB, Sweden) applied to help prevent future migration (Figs 14 and 15). The tibia circular frame had a combination of fixation elements including fine wire and hydroxyapatite-coated half-pins. The half-pins were easily removed as they had not bonded to the bone. The wires proved to be more difficult to extract as the bone seemed to have compressed them. The patient's function had returned to his preinjury state at 1-year follow-up.

The final range of motion was observed, with a maximum pronation of $25^{\circ}$ and supination of $60^{\circ}$ (Fig. 16). According to the patient, this matched his preinjury range of motion and he was satisfied with the overall outcome (Fig. 17).

\section{Case 3}

A 58-year-old lady with osteopetrosis was hit by a car and sustained a closed transverse fracture to her right tibia. She had previously experienced multiple fractures from low-energy trauma, which had been treated with a variety of techniques. After a detailed discussion, the patient elected to undergo circular frame treatment to her tibia but with a flexible nail inserted to help reduce her frame time by increasing construct stiffness. After 6 months, she developed severe sepsis in her left total hip replacement. This required intensive care admission and respiratory support. 

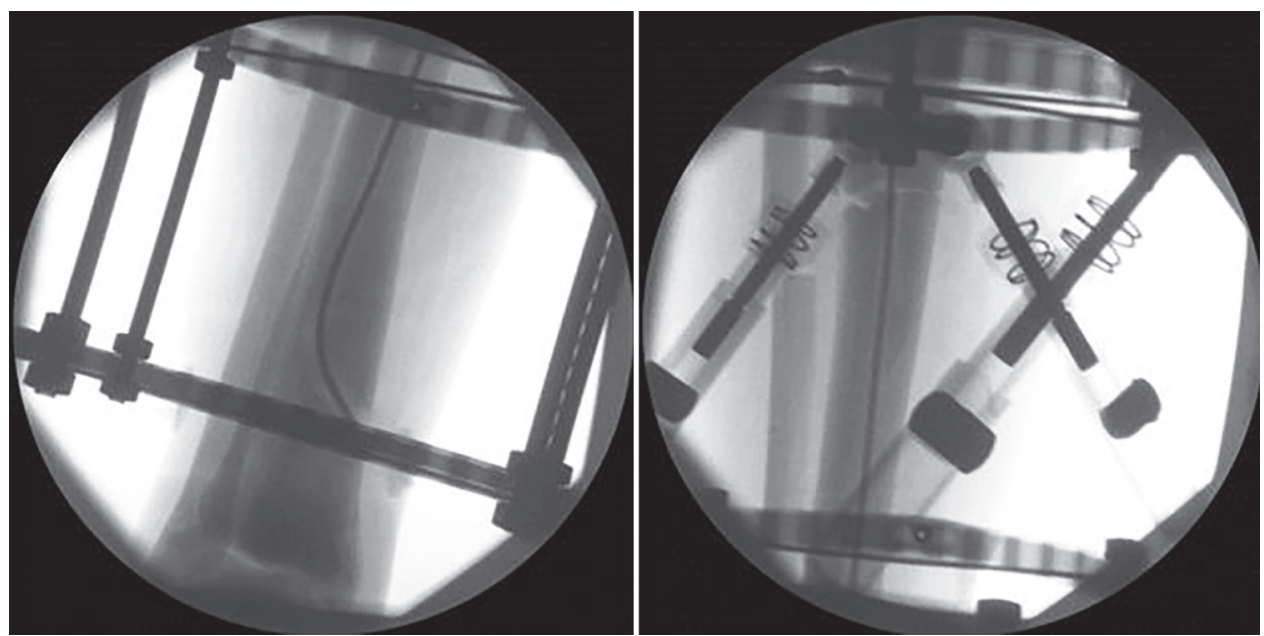

Fig. 18: Intraoperative radiograph showing intramedullary titanium elastic nail and circular frame

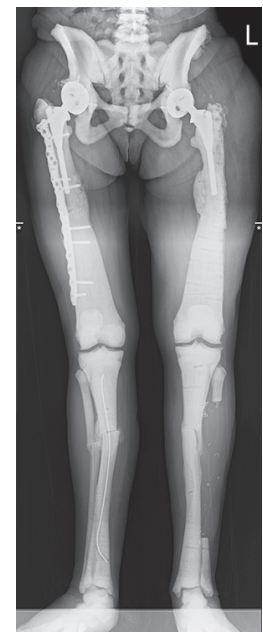

Fig. 19: Long leg alignment radiograph shows a healing right tibia 9 months after surgery

She had experienced several mild pin-site infections in her right tibia and had a long-standing chronic sinus from mandible osteonecrosis. The left hip sepsis was treated and the frame was removed from the right tibia. The callus which had formed around the right tibia in combination with the flexible nail gave enough mechanical support for the fracture to continue healing without the circular frame.

After creating an intramedullary canal utilising the drilling technique and describing the key factors, a titanium elastic nail was able to be inserted from a distal opening in the tibia. The construct was further stabilised with a circular frame around the tibia to control rotation and allow early weight-bearing (Fig. 18).

At 9-month follow-up, some of the lower limb alignment has been lost into valgus at the fracture site (Fig. 19). This position has been accepted, given the problems with her previous episode of sepsis, her resolution of pain, and a return to her preinjury function.

\section{Case 4}

A 40-year-old lady with pycnodysostosis presented following a fall from standing height. She had increasing symptoms of pain from a 16-month-old nonunion of a proximal ulna fracture and

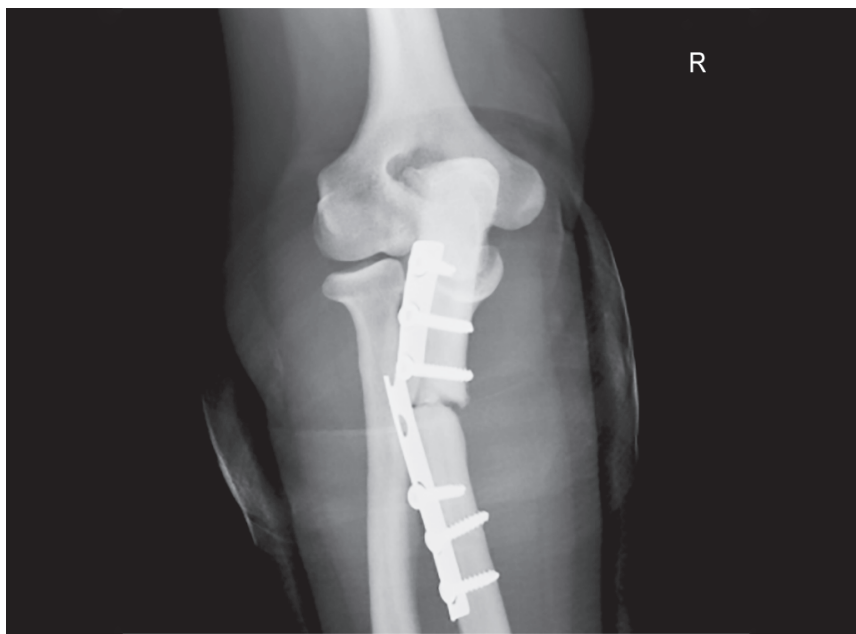

Fig. 20: Radiograph highlighting a proximal ulna nonunion and broken metal ware. The proximal ulna fracture shows no evidence of union, with translation and angulation at the fracture site. The metalwork has failed through fatigue before union could be achieved

broken metalwork (Fig. 20). An operation was planned to correct her resulting forearm deformity, improve her function, and relieve her pain. The broken plate and screws were removed through the previous posterior scar. There was no intramedullary canal, so a new one was created (Fig. 21). During the procedure, it became difficult to create a canal distal to the nonunion site due to the small diameter of the bone and the previous deformity. As such a corrective osteotomy was performed and the distal segment sequentially drilled to create a canal (Figs 22 and 23). The whole construct was fixed with a titanium intramedullary ulna nail (Acumed, Hillsboro, OR, USA) (Fig. 24). Due to the patient's short stature, the nail had to be shortened using bolt cutters. The forearm rotation was corrected and placed into an above elbow backslab (Fig. 25). The most ideal position to cast the forearm is in full supination as this stretches out the intraosseous membrane and will help improve rehabilitation. Per our department protocol for nonunion surgery, a series of sterile tissue specimens are taken at the time of surgery to rule out an infective component of the nonunion. No infection was found in this case and the patient was doing well at her 6-week follow-up. 


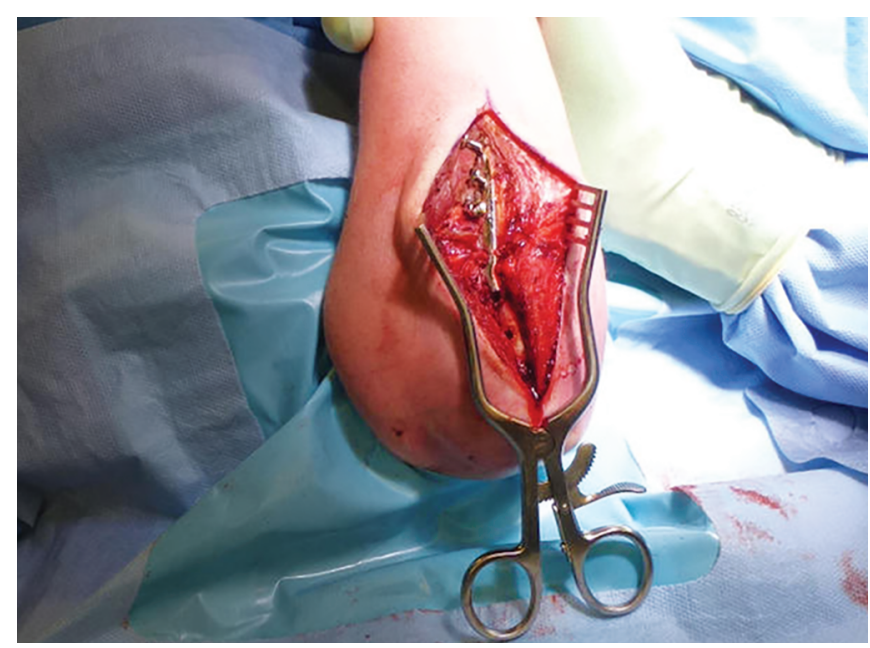

Fig. 21: The proximal ulna nonunion and broken metalwork is exposed

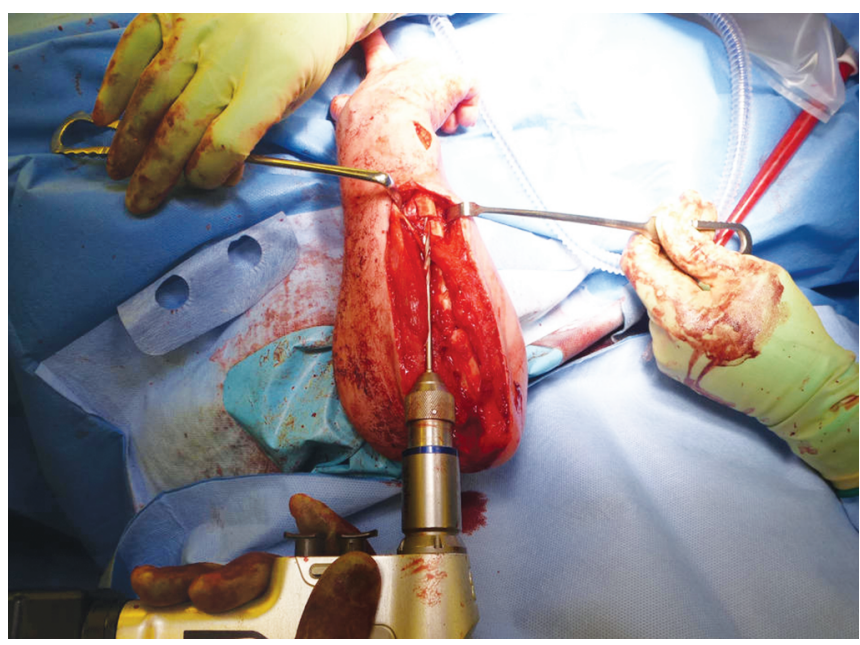

Fig. 22: A $3.2 \mathrm{~mm}$ drill is being used to create a canal in the distal segment - note the small size of the bone and no intramedullary cavity. An osteotomy was required to access the smallest cross section of the ulna and carefully create the canal

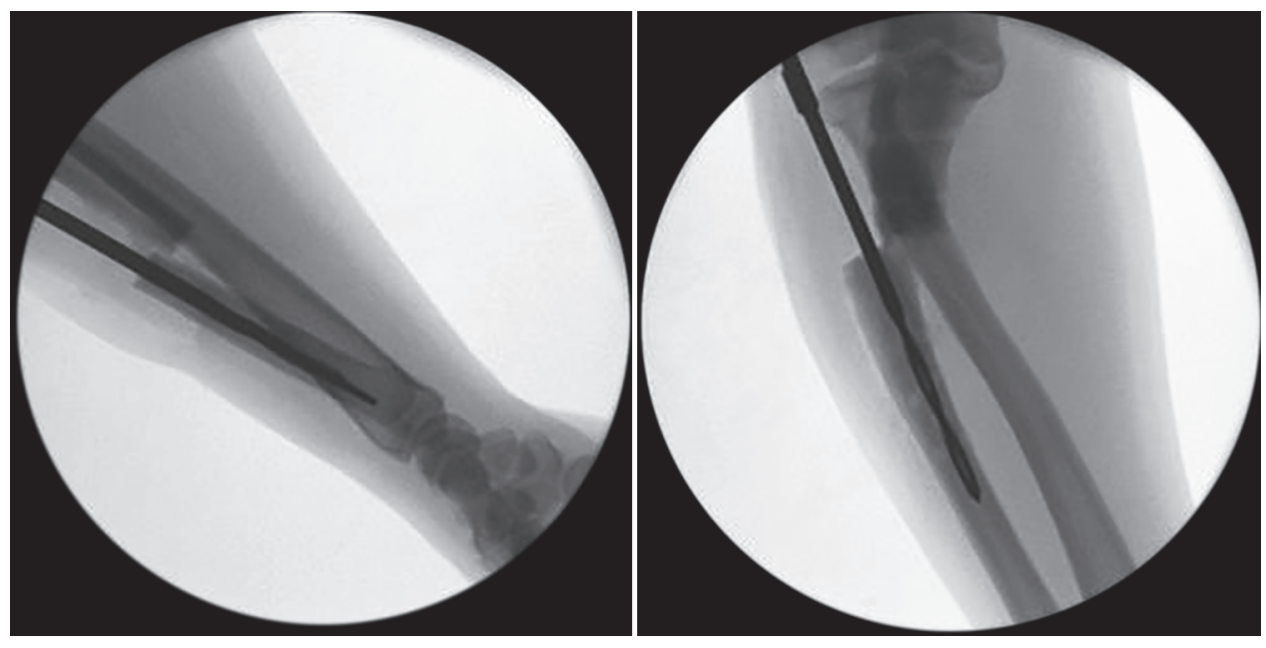

Fig. 23: Orthogonal radiographs depicting careful drilling from proximal to distal, before and after the osteotomy

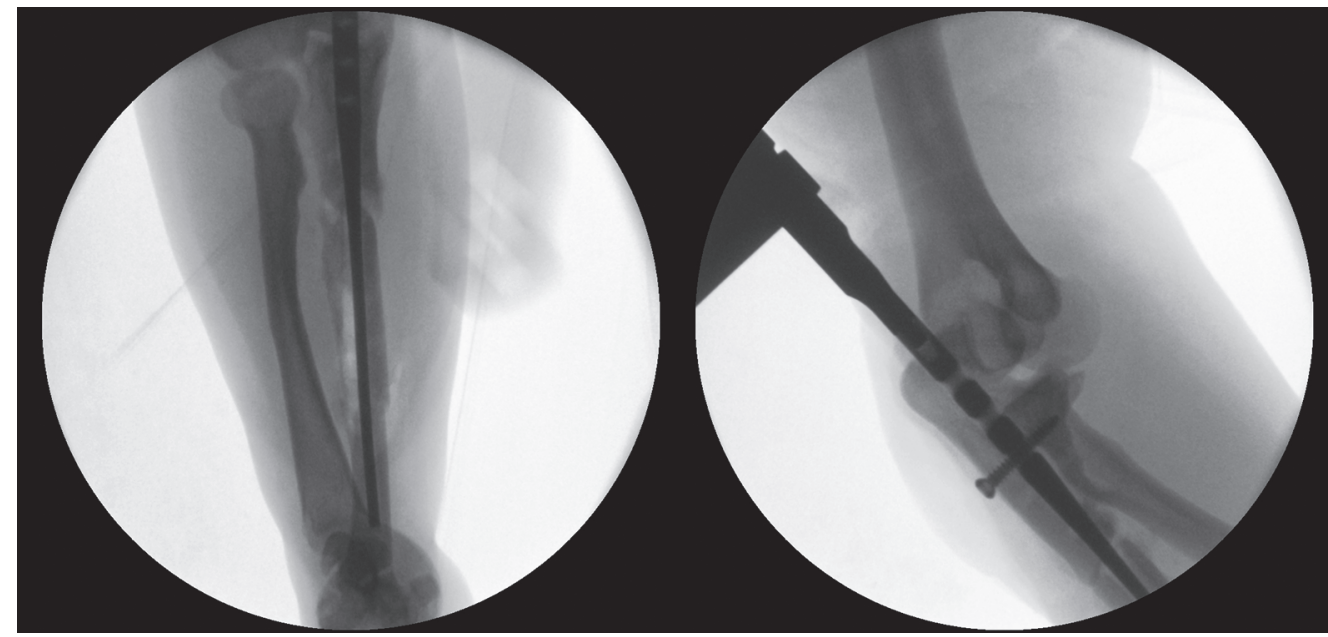

Fig. 24: Insertion of the titanium ulna nail with proximal locking 


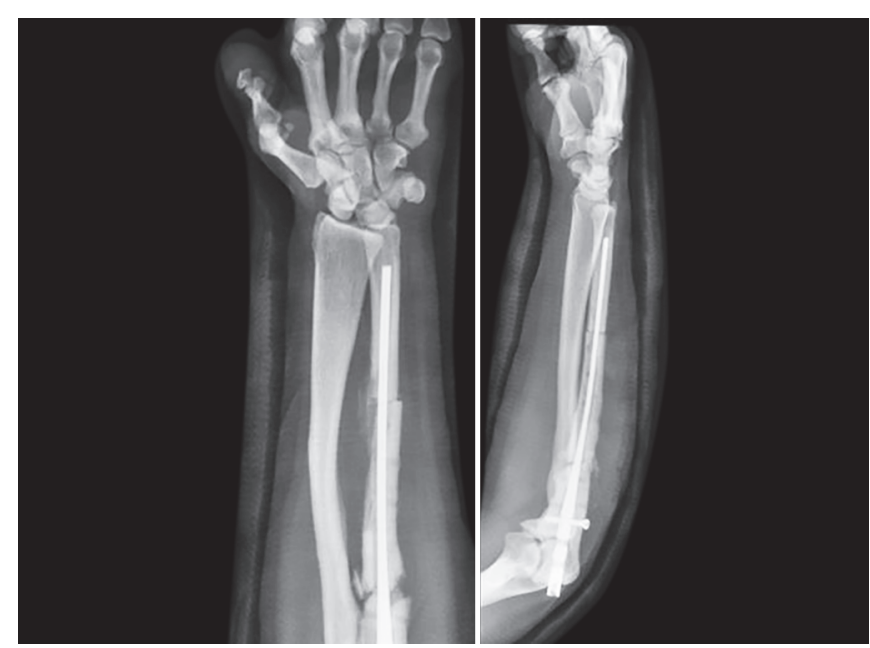

Fig. 25: Postoperative radiographs 4 weeks following surgery. Note the corrective osteotomy

\section{Conclusion}

Long-bone fractures in patients with osteopetrosis are difficult to manage and are technically demanding. By detailing this technique, an intramedullary option is now available for surgeons when deciding upon ideal fracture fixation method. The creation of an intramedullary canal allows intramedullary fracture fixation by a variety of different devices as detailed.
In our experience, adhering to the detailed key factors is essential for success. The technique does not require any specialised equipment and has a broad range of application, including acute fracture fixation and treatment of nonunion.

\section{References}

1. Stark Z, Savarirayan R. Osteopetrosis. Orphanet J Rare Dis 2009;4:5. DOI: 10.1186/1750-1172-4-5.

2. Beighton $\mathrm{P}$, Hamersma $\mathrm{H}, \mathrm{Cremin} B J$. Osteopetrosis in South Africa. The benign, lethal and intermediate forms. S Afr Med J 1979;55(17):659-665.

3. Shapiro F. Osteopetrosis. Current clinical considerations. Clin Orthop Relat Res 1993;294:34-44. DOI: 10.1097/00003086-199309000-00005.

4. Del Fattore A, Cappariello A, Teti A. Genetics, pathogenesis and complications of osteopetrosis. Bone 2008;42(1):19-29. DOI: 10.1016/ j.bone.2007.08.029.

5. Huang J, Pan J, Xu M, et al. Successful open reduction and internal fixation for displaced femoral fracture in a patient with osteopetrosis: case report and lessons learned. Medicine (Baltimore) 2017;96(33):e7777. DOI: 10.1097/MD.0000000000007777.

6. Blank R, Bhargava A, . Osteopetrosis; 2017, eMedicine available at: https://emedicine.medscape.com/article/123968-overview $\#$ \#7.

7. Saftig $\mathrm{P}$, Hunziker $\mathrm{E}$, Wehmeyer $\mathrm{O}$, et al. Impaired osteoclastic bone resorption leads to osteopetrosis in cathepsin-K-deficient mice. Proc Natl Acad Sci U S A 1998;95(23):13453-13458. DOI: 10.1073/ pnas.95.23.13453.

8. de Palma L, Tulli A, Maccauro G, et al. Fracture callus in osteopetrosis. Clin Orthop Relat Res 1994;308:85-89. DOI: 10.1097/00003086199411000-00014. 